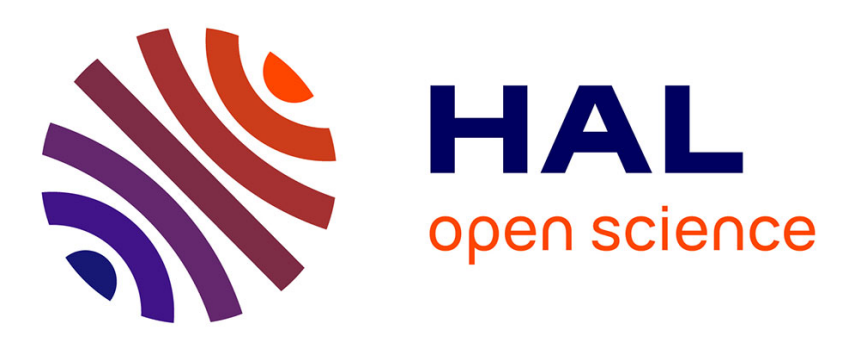

\title{
Le nouvel intérêt des villes intermédiaires pour les terres agricoles : actions foncières et relocalisation alimentaire
}

Adrien Baysse-Lainé, Coline Perrin, Claire Delfosse

\section{To cite this version:}

Adrien Baysse-Lainé, Coline Perrin, Claire Delfosse. Le nouvel intérêt des villes intermédiaires pour les terres agricoles : actions foncières et relocalisation alimentaire. Géocarrefour - Revue de géographie de Lyon, 2018, 92 (4), pp.1-21. 10.4000/geocarrefour.10417 . hal-01862341

\section{HAL Id: hal-01862341 \\ https://hal.science/hal-01862341}

Submitted on 25 Oct 2018

HAL is a multi-disciplinary open access archive for the deposit and dissemination of scientific research documents, whether they are published or not. The documents may come from teaching and research institutions in France or abroad, or from public or private research centers.
L'archive ouverte pluridisciplinaire HAL, est destinée au dépôt et à la diffusion de documents scientifiques de niveau recherche, publiés ou non, émanant des établissements d'enseignement et de recherche français ou étrangers, des laboratoires publics ou privés. 


\section{Géocarrefour}

$92 / 4 \mid 2018$

Varia

\section{Le nouvel intérêt des villes intermédiaires pour les terres agricoles : actions foncières et relocalisation alimentaire}

The new interest of intermediate cities for agricultural land: land actions and food relocation

Adrien Baysse-Lainé, Coline Perrin and Claire Delfosse

\section{OpenEdition}

\section{Journals}

\section{Electronic version}

URL: http://journals.openedition.org/geocarrefour/10417

ISSN: 1960-601X

Publisher

Association des amis de la Revue de géographie de Lyon

Brought to you by Université Lumière Lyon 2

\section{UNR-RA}

Electronic reference

Adrien Baysse-Lainé, Coline Perrin and Claire Delfosse, "Le nouvel intérêt des villes intermédiaires pour les terres agricoles : actions foncières et relocalisation alimentaire », Géocarrefour [Online], 92/4 | 2018, Online since 11 May 2018, connection on 12 May 2018. URL : http:// journals.openedition.org/geocarrefour/10417

This text was automatically generated on 12 May 2018

(C) Géocarrefour 


\title{
Le nouvel intérêt des villes intermédiaires pour les terres agricoles : actions foncières et relocalisation alimentaire
}

\author{
The new interest of intermediate cities for agricultural land: land actions and \\ food relocation
}

Adrien Baysse-Lainé, Coline Perrin and Claire Delfosse

En avril 2016, la commune d'Albi annonçait son souhait de préempter 73 ha de terres agricoles dans la plaine du Tarn voisine de la ville, pour y installer des maraîchers (Carcenac, 2016). Cette mobilisation de terres agricoles par une collectivité urbaine est destinée à assurer l'approvisionnement de la ville dans une perspective «locavore " (Poulot, 2012): les maraîchers doivent s'engager à commercialiser leur production en circuit court de proximité. Comme Albi, de nombreuses collectivités urbaines françaises portent depuis une dizaine d'années un intérêt nouveau aux terres agricoles de leur périphérie. Auparavant perçues comme multifonctionnelles (Duvernoy et al., 2005), en lien avec des préoccupations paysagères et environnementales (Thareau et Fabry, 2013), ces terres sont désormais appréhendées comme la base potentielle de systèmes alimentaires urbains relocalisés. Ce regain d'intérêt doit être relié à l'évolution des représentations du foncier agricole, depuis "une vision productive [...] à une vision marchande (bien), puis à une valeur 'brute' (ressource) et enfin depuis peu 'éthique' (alimentation)» (Margetic et Chaucesse, 2012). Il doit aussi être remis dans le contexte juridique national favorable à la protection des terres agricoles et à l'approvisionnement local de la restauration collective (lois « Grenelle » de 2009 et 2010), ainsi qu'aux projets alimentaires territoriaux et à l'agroécologie (loi d'avenir agricole de 2014).

2 Comme le soulignent Jarrige et Napoleone (2015), ce nouvel intérêt des collectivités urbaines pour le foncier agricole a surtout été étudié, en France, dans les grandes villes 
métropolisées. Ainsi, l'Agence des espaces verts francilienne «est devenue le maître d'œuvre de la relocalisation maraîchère [...] biologique » (Poulot, 2014), elle installe des arboriculteurs et soutient les industries de première transformation (Guiomar, 2014). Des communes et un syndicat mixte de la Métropole de Lyon acquièrent des terres pour développer des circuits courts de proximité (Brand, 2015). Lille Métropole a créé ex nihilo une zone maraîchère, arboricole et horticole biologique où elle a installé huit agriculteurs (Margetic et al., 2017). Toulouse Métropole a acheté du foncier pour maintenir une exploitation maraîchère biologique (Duvernoy et Paradis, 2016). À Montpellier, des terres publiques ont été à deux reprises allouées à des agriculteurs (Jarrige et Perrin, 2017).

3 La prise en compte des questions agricoles et alimentaires est plus récente dans les villes intermédiaires. Ces villes sont d'abord celles qui occupent des positions secondaires dans la hiérarchie urbaine : trop bas pour connaître des processus de métropolisation, elles sont suffisamment haut pour polariser un arrière-pays. Elles forment à ce titre les « pôles urbains de l'espace non métropolisé » (Carrier et Demazière, 2012). Les villes intermédiaires sont ensuite des relais entre les métropoles et les espaces ruraux. Leur rôle de pivot villes-campagnes est souvent minoré (Rieutort, 2018) : les métropoles ont longtemps concentré l'intérêt des études urbaines, qui en ont tiré leurs modèles, souvent érigés en normes (Demazière et al., 2012).

4 Les collectivités des villes intermédiaires ont un positionnement parfois ambigu sur l'artificialisation. Elles traduisent souvent leur volonté de développement par une croissance du bâti économique aux franges urbaines (Arnal, 2012a). Des travaux montrent toutefois qu'elles développent aussi des stratégies agricoles ou alimentaires (Marty, 2014), par exemple autour des produits de terroir (Arnal, 2012b; Delfosse, 2011 et 2015). Les actions foncières de ces collectivités en lien avec un projet alimentaire se multiplient : Léger-Bosch (2015) en identifie par exemple à Bourgoin-Jallieu, Voiron (Isère), Riom, au Puy-en-Velay ou en Haute-Savoie. Elles peuvent être classées en quatre types (Terre de Liens, 2013): (i) la location de terres publiques, (ii) la location de fermes (terres et bâtiments) publiques, (iii) la location-vente de fermes, alors appelées «ferme-relais » et (iv) l'exploitation en régie publique.

$5 \quad$ L'objectif de cet article est de comprendre pourquoi et comment les collectivités des villes intermédiaires interviennent sur le foncier agricole pour relocaliser leur système alimentaire. Il s'agit notamment d'explorer leur éventuelle spécificité par rapport aux métropoles. Pour ce faire, nous analysons des mises à disposition de terres ou de fermes par les intercommunalités d'Amiens, Millau et Saint-Affrique. Quelles trajectoires débouchent sur ces actions foncières visant la relocalisation? Ciblent-elles certaines catégories de terres et d'agriculteurs? Dans quelle mesure le statut (tant juridique que symbolique) de ces terres évolue-t-il lors des opérations?

6 Pour répondre à ces questions, nous donnons d'abord un aperçu des trajectoires d'actions foncières des trois intercommunalités. Nous revenons ensuite sur la manière dont elles sélectionnent certains agriculteurs, ciblent certains modèles agricoles et cherchent à les implanter sur des terres aux statuts physique et juridique particuliers. Enfin, nous montrons comment ces mises à disposition de terres publiques constituent des constructions incomplètes du foncier comme ressource nourricière de proximité. 


\section{Terrains et méthodologie}

7 Notre propos est une analyse croisée d'enquêtes réalisées dans les intercommunalités de trois villes intermédiaires. Les terrains ont été choisis selon la méthode des cas contrastés, pour maximiser la diversité des observations. Il s'agit de mettre en lumière d'éventuels invariants, non dépendants des contextes territoriaux. Deux zones d'étude ont été sélectionnées pour leur représentativité de la variété des six «grands ensembles structurels d'exploitations agricoles » définis par Levesque et al. (2011) : le Bassin parisien et le Nord et l'Est de la France d'un côté, le piémont pyrénéen, le cœur du Massif central et le Grand Ouest de l'autre côté. En leur sein, nous avons sélectionné deux zones d'agriculture spécialisée et organisée par les circuits longs, dans des productions différentes : grandes cultures dans la plaine picarde et élevage ovin dans le sud du Massif central. Nous avons enfin ciblé trois villes intermédiaires de taille différente, dont les collectivités mettent en œuvre des actions foncières pour la relocalisation alimentaire.

8 Ainsi, Amiens Métropole (180 $000 \mathrm{~h}$, carte 1$)$ s'étend à la fois sur les plateaux picards - de larges étendues de terres planes $(50-150 \mathrm{~m})$ très fertiles où s'est développée une céréaliculture intensive et exportatrice - et sur des fonds de vallées humides - partagés entre espaces naturels et agricoles (pâturage et maraîchage). Le fermage y est majoritaire, la propriété est dispersée par rapport à l'exploitation, les sociétés agricoles sont courantes et les agriculteurs très présents sur le marché foncier (Levesque et al., 2011). Marquée par des logiques entrepreneuriales, l'agriculture entretient peu de liens avec la ville. Les intercommunalités de Millau Grands Causses (29000 h, carte 2) et du SaintAffricain (13000 h, carte 2) font partie des moyennes montagnes du sud du Massif central, où les plateaux calcaires (atteignant $850 \mathrm{~m}$ ) côtoient des gorges et des plaines vallonnées (autour de $300 \mathrm{~m}$ d'altitude). L'élevage ovin laitier pour les industriels de Roquefort est la principale production. Le fermage et le phénomène sociétaire y sont en progression, le marché foncier est ouvert et le bâti rural dispersé. Les circuits courts sont en forte croissance, portés par la diversité des produits locaux et l'émergence de collectifs de producteurs.

9 Malgré leur différence de taille, Amiens et Millau sont des villes secondaires de « systèmes urbains de proximité » (Berroir et al., 2017) qui ont à leur tête des métropoles à l'échelle nationale: Paris et Montpellier (les influences respectives de Lille et Rodez étant limitées). Saint-Affrique n'apparaît pas dans cette étude, mais Férerol (2013) montre que - même s'il s'agit d'une «petite ville inférieure de niveau 1 »- sa zone d'influence principale s'étend sur plus de $1000 \mathrm{~km}^{2}$ du Sud-Aveyron. Elle n'y est que marginalement concurrencée par Albi et Rodez. 
Cartes 1 (en haut à gauche) et 2 (en bas à droite) : Situations des études de cas dans les zones d'études

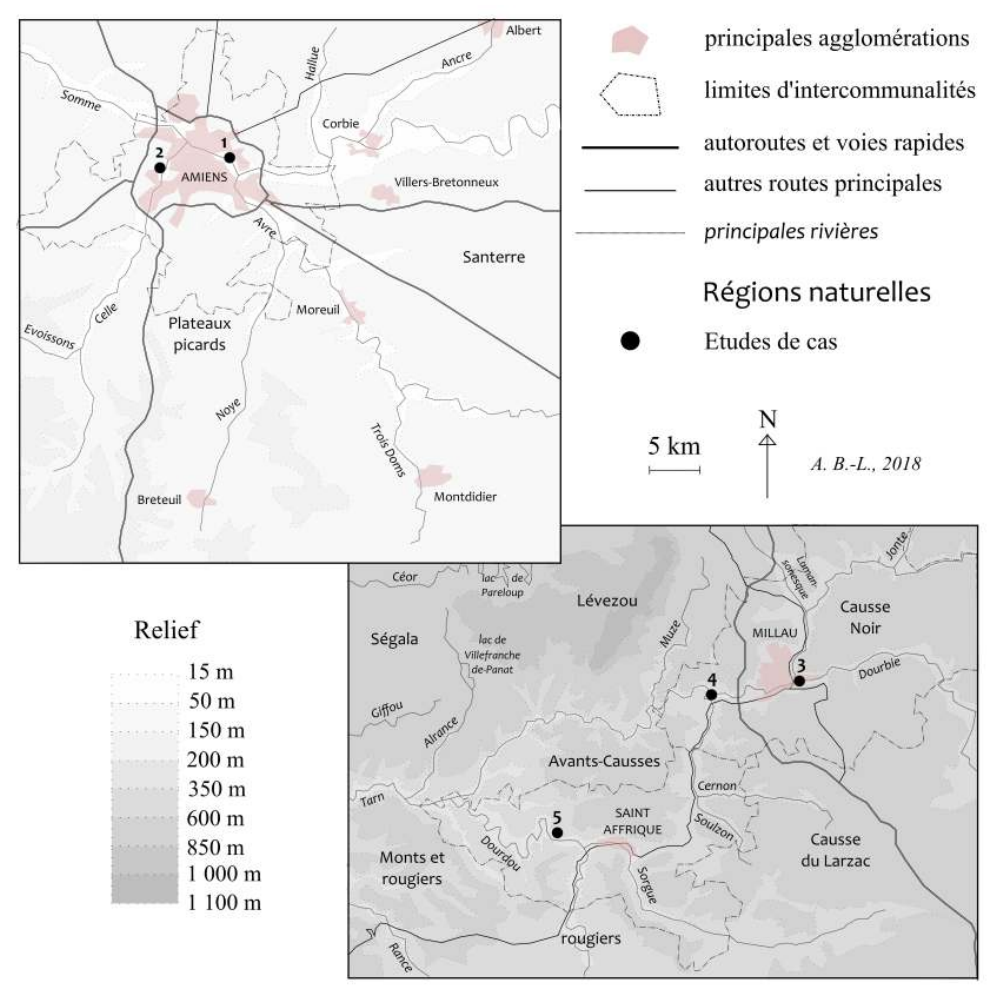

10

Dans ces trois intercommunalités, nous avons étudié l'exhaustivité des mises à disposition de foncier public à des exploitations visant une commercialisation locale. Nous avons conduit vingt-cinq entretiens semi-directifs avec tous les types d'acteurs ayant participé aux projets : les agriculteurs bénéficiaires, les élus communaux et intercommunaux en charge de l'agriculture et/ou de l'alimentation, des $\mathrm{DGS}^{1}$, chefs de pôle, chargés de mission et techniciens d'intercommunalités, des conseillers fonciers Safer ${ }^{2}$, des chargés de mission de Chambres d'agriculture et un animateur régional du mouvement Terre de Liens. Nous avons également analysé des comptes rendus de conseils municipaux et communautaires, des archives personnelles d'enquêtés et des arrêtés municipaux.

Le tableau 1 donne un aperçu des études de cas. Il s'agit de projets réalisés depuis 2010, sauf un programme mené par Amiens Métropole dans les années 1990 et 2000. Les surfaces sont plutôt limitées ( 1,2 à 11,0 ha), du fait de l'orientation maraîchère choisie. L'organisation du travail est très variée: on trouve des exploitations individuelles (familiales ou non) avec un à deux actifs, mais aussi des exploitations à caractère collectif : GAEC à deux associés, atelier-chantier d'insertion employant une quinzaine de salariés temporaires. L'espace-test (une couveuse d'exploitations agricoles permettant aux porteurs de projet dépourvus de foncier de débuter leur activité en attendant d'accéder à la terre) apparaît comme une forme hybride de responsabilité individuelle et de solidarité collective. Ces exploitations ont souvent été créées par leur exploitant actuel (lors d'une reconversion professionnelle), une s'est agrandie et d'autres simplement maintenues. Les terres sont à la fois des friches remises en culture et du foncier déjà agricole dont l'usage a été modifié. Enfin, les modes de commercialisation couvrent une large part du spectre des circuits courts, aussi bien traditionnels (marché, vente à la ferme) que plus récents (paniers, AMAP, magasin de producteurs, restauration collective). 
Les cartes 1 et 2 montrent que les études de cas sont situées sur la frange urbaine à Amiens tandis qu'elles sont plutôt localisées dans l'espace rural dans le Sud-Aveyron. Chaque commune-centre accueille toutefois une étude de cas, étant donné la grande étendue de Millau $\left(168 \mathrm{~km}^{2}\right)$ et Saint-Affrique $\left(111 \mathrm{~km}^{2}\right)$. Toutes se trouvent près de cours d'eau importants: la Somme (et son affluent la Celle) dans l'Amiénois, le Tarn (et son affluent le Dourdou) dans le Sud-Aveyron, en fond de vallée ou sur le rebord du premier coteau.

Tableau 1 : Description de l'échantillon

\begin{tabular}{|c|c|c|c|c|c|}
\hline \multirow{2}{*}{$\begin{array}{c}\text { Terrain } \\
\begin{array}{c}\text { Date de réalisation du } \\
\text { projet }\end{array} \\
\end{array}$} & \multicolumn{2}{|c|}{ communauté d'agglomération Amiens Métropole } & \multicolumn{2}{|c|}{ communauté de communes Millau Grands Causes } & \multirow{2}{*}{\begin{tabular}{|c|}
$\begin{array}{c}\text { communauté de communes } \\
\text { du Saint-Affricain }\end{array}$ \\
$2008-2016$ \\
\end{tabular}} \\
\hline & années 1990 et 2000 & 2010-2013 & 2010-2011 & 2013-2016 & \\
\hline $\begin{array}{l}\text { Exploitations } \\
\text { bénéficiaires }\end{array}$ & $\begin{array}{l}\text { sept exploitations } \\
\text { individuelles }\end{array}$ & un GAEC & $\begin{array}{l}\text { un atelier-chantier } \\
\text { d'insertion associatif }\end{array}$ & une exploitation individuelle & un espace-test \\
\hline Production & Maraîchage conventionnel & \multicolumn{4}{|c|}{ Maraichage biologic } \\
\hline Commercialisation & $\begin{array}{l}\text { Marchés et grande } \\
\text { distribution (à Amiens) }\end{array}$ & $\begin{array}{c}\text { AMAP, paniers, RHD et vente à } \\
\text { la ferme (à Amiens) }\end{array}$ & \begin{tabular}{|} 
Paniers, marché, cuisine \\
centrale (à Millau)
\end{tabular} & $\begin{array}{l}\text { Marché de producteurs (à } \\
\text { Millau) }\end{array}$ & Marché (à Saint-Affrique) \\
\hline Surface & 6,5 ha en propriété publique & 11 ha & $\begin{array}{l}8 \text { ha (dont } 5,5 \text { ha en } \\
\text { propriété publique) }\end{array}$ & 1,2 ha & 4,5 ha \\
\hline Actifs & environ 15 & 3 & 18 & 1 & 3 \\
\hline $\begin{array}{l}\text { Parcours et capitaux } \\
\text { des agriculteurs } \\
\text { bénéficiaires }\end{array}$ & $\begin{array}{l}\text { Issus d'un groupe dominé } \\
\text { (économiquement et } \\
\text { symboliquement) du milieu } \\
\text { agricole (les « hortillons »): } \\
\text { transmissions familiales. }\end{array}$ & \begin{tabular}{|} 
Reconversions \\
professionnelles, dotation \\
importante en capital culturel \\
(fils d'éleveurs biologiques \\
ancien président d'un réseau \\
d'AMAP, conseiller technique \\
maraîchage dans un GRAB)
\end{tabular} & \begin{tabular}{|c|} 
Fondateur : forte dotation \\
en capital social, issu du \\
milieu agricole et de la \\
commune. Employés: \\
faibles dotations en capital \\
économique, en \\
reconversions \\
professionnelles.
\end{tabular} & $\begin{array}{c}\text { Reconversion professionnelle } \\
\text { puis salariat agricole } \\
\text { localement pendant } 15 \text { ans. } \\
\text { Faible dotation en capital } \\
\text { économique. }\end{array}$ & $\begin{array}{c}\text { Reconversions } \\
\text { professionnelles, avec } \\
\text { formation au lycée agricole de } \\
\text { Saint-Affrique. }\end{array}$ \\
\hline $\begin{array}{l}\text { Trajectoire de } \\
\text { l'exploitation }\end{array}$ & $\begin{array}{l}\text { Maintien (avec changement } \\
\text { de propriétaire) ou } \\
\text { agrandissement }\end{array}$ & Création & Agrandissement & Création & Création \\
\hline Trajectoire des terres & $\begin{array}{c}\text { Maintien ou remise en } \\
\text { culture }\end{array}$ & $\begin{array}{l}\text { Changement de production } \\
\text { (depuis la céréaliculture) }\end{array}$ & $\begin{array}{c}\begin{array}{c}\text { Remise en culture d'une } \\
\text { friche }\end{array} \\
\end{array}$ & \begin{tabular}{|c|} 
Changement de production \\
(depuis une prairie)
\end{tabular} & $\begin{array}{l}\text { Changement de production } \\
\text { (depuis une prairie) }\end{array}$ \\
\hline $\begin{array}{l}\text { Numéro de report sur les } \\
\text { cartes }\end{array}$ & 1 & 2 & 3 & 4 & 5 \\
\hline
\end{tabular}

Notes : GAEC = Groupement agricole d'exploitation en commun ; AMAP = Association pour le maintien d'une agriculture paysanne; $\mathrm{GRAB}=$ Groupement régional d'agriculteurs biologiques ; RHD =

Restauration hors domicile.

\section{Intercommunalités et actions foncières agricoles : trois exemples de trajectoires}

Les trois intercommunalités choisies illustrent trois cheminements vers des actions foncières agricoles, avec des entrées respectivement patrimoniale puis économique à Amiens, sanitaire puis nutritionnelle à Millau et liée à la formation et l'emploi à SaintAffrique.

\section{Patrimonialisation de l'agriculture intra-urbaine et installation de maraîchers périurbains à Amiens}

14 À la fin des années 1980, l'intercommunalité « commence à se préoccuper sérieusement du déclin de l'activité maraîchère dans les hortillonnages $»^{3}$. Ces marais situés près du centre ancien et aménagés en petites îles maraîchères tendent à se transformer en jardins privatifs. Le paysage se ferme. Un plan de sauvegarde est rédigé en 1997. La collectivité "décide d'acheter des terrains pour les mettre en location: avec le développement de l'activité de loisir, les prix fonciers augmentaient et pour les hortillons, il était difficile d'accéder à la terre $»^{1}$. Les parcelles les plus propices au maraîchage (proches des routes et peu en friche) sont ciblées. Sur les 12,6 ha acquis, 6,5 sont loués à sept maraîchers déjà en activité, dont la majorité exploite d'autres terres. L'intercommunalité les accompagne 
pour commercialiser leurs légumes sous une marque locale, notamment dans la grande distribution.

Tandis que la patrimonialisation des hortillonnages participe de la politique d'attractivité (notamment touristique) de la ville, les espaces agricoles périurbains sont plutôt voués à l'artificialisation, la politique de développement économique intercommunale étant fondée sur les zones commerciales et d'activités. En 2010, un maraîcher cherche du foncier pour s'installer. L'intercommunalité lui propose des parcelles partiellement en propriété publique, situées sur un champ de captage d'eau potable dans le périmètre d'une $\mathrm{ZAC}^{4}$ non réalisée. Comme l'explique une chargée de mission, "c'était une opportunité, l'occasion a fait le larron. [...] Ça nous va bien, parce qu'au moins, les terrains sont utilisés. [...] Mais ça reste très anecdotique, comme projet ». Le maraîcher et un associé s'y installent en 2013, accompagnés par Terre de Liens et soutenus par deux élus du syndicat d'aménagement de la ZAC (dont son président). Ces derniers sont par ailleurs respectivement (i) vice-président d'Amiens Métropole, voisin du porteur de projet et ancien enseignant de l'animateur régional de Terre de Liens et (ii) conseiller général et membre de Terre de Liens. Malgré l'étendue de foncier disponible répondant aux mêmes critères, aucun autre projet d'installation n'est envisagé. Lors des élections de 2014, les élus de gauche cèdent la place à une équipe orientée à droite, pour laquelle de tels projets ne sont pas une priorité.

Amiens Métropole a donc d'abord mené un programme foncier de protection d'un patrimoine environnemental. Il a permis de maintenir une forme traditionnelle de maraîchage urbain professionnel, qui a disparu ailleurs faute d'action foncière notamment à Bourges (Gallien, 2011). Un projet ponctuel d'installation, impulsé par le porteur de projet agricole et fortement soutenu par Terre de Liens, a ensuite répondu à des enjeux de développement économique.

\section{Un programme foncier éphémère à Millau}

17 À Millau, une double traduction - de la santé à l'alimentation puis de l'alimentation au foncier - a été opérée à l'initiative de la première vice-présidente de la communauté de communes, également première adjointe au maire. En 2008, la nouvelle équipe élue, marquée à gauche, décide d'agir sur l'état sanitaire de la population via l'alimentation. La cuisine centrale est considérée comme un levier : ses approvisionnements et menus sont restructurés pour intégrer des aliments issus de l'agriculture biologique. Le conseil municipal souhaite éviter l'achat de produits lointains et se tourne donc vers les producteurs locaux. Il constate " qu'en viande et en laitages, il y a à peu près ce qu'il faut, mais qu'en maraîchage, ce n'est pas évident $»^{3}$ et souhaite "protéger le foncier pour développer le maraîchage bio. $»^{5}$

L'action foncière n'a pas été une initiative publique : elle est venue d'un atelier-chantier d'insertion associatif, le Jardin du Chayran, principal producteur local de légumes biologiques. Cette exploitation ne disposait pas d'assez de foncier pour répondre à une demande croissante de paniers. En 2010, elle cible un terrain de 5,5 ha en friche depuis trois ans, acheté par l'intercommunalité avec le produit de la taxe départementale pour les espaces naturels sensibles. Le sol alluvionnaire est bien adapté au maraîchage. L'association " a demandé à la collectivité de pouvoir cultiver : ça lui a été refusé à cause du statut d'espace naturel sensible [...]. Son président a donc organisé un happening, une manifestation à la José Bové $»^{3}$. Vétéran de la lutte du Larzac ${ }^{6}$, le président remobilise son 
capital militant en organisant l'occupation des terres, qui sont labourées par des agriculteurs et ensemencées par des bénévoles. L'association est soutenue par plusieurs élus (dont la première vice-présidente) pour ses rôles alimentaire et de réinsertion sociale. Elle obtient en 2011 des droits de culture.

Après avoir mis à disposition cette réserve foncière, la communauté de communes décide d'acquérir d'autres terres pour développer l'offre locale en légumes biologiques, dans le cadre de sa politique de développement économique et de soutien aux filières locales. La mise en œuvre de ce projet est néanmoins limitée car, en ciblant les terres des étroits fonds de vallée, elle entre en concurrence avec les éleveurs. Ainsi, seuls 1,2 ha ont été acquis. Un maraîcher, sélectionné par le président du Jardin du Chayran, y a créé son exploitation. Les élus les plus engagés dans cette politique n'ont pas été réélus en 2014.

20 À Millau, un programme foncier de développement d'une filière de maraîchage biologique a émergé au croisement (i) de revendications agricoles d'accès au foncier et (ii) d'une volonté politique d'amélioration de l'accessibilité sociale à une alimentation saine. Il s'est appuyé sur un acteur agricole à la fois bénéficiaire et sous-traitant de la politique publique.

\section{Création d'un espace-test et mise en place d'une veille foncière à Saint-Affrique}

21 De nombreux maraîchers s'installent dans les vallées entourant Saint-Affrique à partir de 2012. Certains, non issus du territoire, ont suivi la formation pour adultes en maraîchage biologique du lycée agricole local. Dès la fin des années 2000, la communauté de communes a accompagné ces installations, en structurant la commercialisation (le débouché du marché hebdomadaire devenant rapidement trop petit) et en créant un espace-test.

22 Cet espace-test est envisagé sur une réserve foncière de 4 ha, constituée pour une ZAC mais en fait inondable. Durant six ans, le projet est reformulé trois fois : d'abord avec une optique d'insertion sociale, puis dans un esprit coopératif (associant élus, maraîchers et consommateurs), enfin dans une démarche partenariale (associant des élus et des organisations d'enseignement et de développement agricole ou local). Le projet achoppe cependant sur la très faible qualité agronomique des sols et leur exposition à des crues violentes. En 2015, lorsque le lycée agricole agrandit son exploitation, il accepte d'échanger certains de ses nouveaux terrains - de meilleure qualité agronomique - contre la réserve foncière intercommunale. L'espace-test y est créé et accueille en 2016 des futurs maraîchers.

23 Pour pérenniser ces nouveaux emplois et faciliter l'installation des maraîchers à l'issue du test, la collectivité cherche aujourd'hui à acquérir des terrains (avec l'aide de la Safer). Elle prévoit de les leur revendre une fois leur activité établie. Toutefois, en parallèle, elle a souhaité créer une autre zone d'activité sur des terres propices au maraîchage. Face à la protestation menée notamment par la Confédération paysanne, l'intercommunalité envisage dans ce projet la mise à disposition de 2 ha de terres à des maraîchers.

24 À Saint-Affrique, un projet d'espace-test maraîcher a été conduit en associant de nombreux partenaires territoriaux (institutionnels et associatifs), ce qui est original car les espaces-tests sont ailleurs plutôt portés par des associations ${ }^{7}$. 
Ces trois trajectoires apparaissent comme des cheminements menant d'enjeux divers (patrimoine, environnement, développement économique et emploi, nutrition-santé, accessibilité sociale) à une action foncière en faveur des circuits alimentaires de proximité. Elles illustrent toutes une inflexion: aucune des trois collectivités n'était auparavant intervenue dans le domaine foncier. Pourtant, il ne s'agit pas de retournement: ces actions ne sont pas guidées par des stratégies de long terme et rencontrent des points de blocage. Deux positionnements ressortent: soit les intercommunalités répondent à des demandes de porteurs de projet (présentée par Terre de Liens à Amiens, sous la contrainte d'une occupation à Millau et d'un mouvement de protestation à Saint-Affrique), soit elles cherchent à acquérir des terres dans des espaces ciblés (les fonds de vallées à Millau et Saint-Affrique ou les hortillonnages à Amiens). Seule la communauté de communes de Saint-Affrique a été à l'origine d'un projet (l'espace-test maraîcher), qu'elle a mené à bien en huit ans. C'est aussi la seule intercommunalité à avoir connu une continuité politique: les trois trajectoires apparaissent fortement dépendantes de l'engagement de quelques élus. Elles conduisent à des actions foncières, d'ampleur certes limitées, mais favorables à la relocalisation alimentaire, et qui ne sont pas poursuivies, ni remises en cause par les nouvelles majorités.

26 Nous nous proposons d'analyser dans quelle mesure ces trajectoires illustrent une modalité commune du traitement foncier de la question alimentaire par les collectivités.

\section{La construction de la base foncière d'un modèle agricole « relocalisé »}

27 Des régularités apparaissent entre les trois trajectoires dans le modèle agricole ciblé, la sélection des agriculteurs bénéficiaires, le moyen de mobiliser les terres et le statut - tant juridique que symbolique - de ces terres.

\section{Le choix d'un modèle agricole " atypique » : le maraîchage biologique en circuit court de proximité}

Les intercommunalités s'orientent vers un modèle agricole spécifique, souvent qualifié "d'atypique " par nos enquêtés au vu des modèles dominants localement. Il s'agit du maraîchage en circuit court de proximité, le plus souvent biologique. Tous les cas recensés se trouvent être des exploitations maraîchères : les élus cherchent en effet à développer et diversifier une offre locale en légumes très réduite (ce qu'évoque également Guiomar (2011)). Les projets récents sont tous en production biologique, parce que les porteurs de projet l'ont souhaité, mais aussi (à Millau et Saint-Affrique) parce que les élus en ont fait une condition de leur soutien. Les exploitants des hortillonnages, soutenus au cours des années 2000, font exception: Amiens Métropole n'a pas exigé de pratiques spécifiques. Enfin, la commercialisation locale fait l'objet d'un engagement entre les producteurs et les intercommunalités. Toutefois, aucune des trois villes n'a formalisé ce modèle agricole maraîcher local. Seule l'orientation biologique a été contractualisée par des clauses environnementales à Amiens et Millau.

Au-delà de ces trois intercommunalités, le soutien au maraîchage biologique en circuits courts de proximité apparaît comme un horizon d'attente des villes. Léger-Bosch (2015) 
note ainsi une surreprésentation de cette production dans son échantillon de maîtrises foncières publiques agricoles en Auvergne-Rhône-Alpes. La nouvelle légitimité des circuits courts de proximité s'est appuyée sur la « promesse de différence » dont ils sont porteurs: nouveau paradigme de développement agricole, réponse aux aberrations de l'agro-industrie et sortie de l'économie de marché (Le Velly, 2017). Leur « consécration institutionnelle » a résulté de la rencontre d'intérêts portés par des chercheurs (experts pour le Ministère de l'Agriculture), des collectivités locales et des associations militantes (Paranthoën, 2015). Ainsi, les circuits courts relèvent d'un «nouvel idéal de la société concernant l'agriculture », particulièrement " relayé par les collectivités et élus » (LégerBosch et Alavoine-Mornas, 2013). Par ailleurs, leur encadrement par les acteurs publics a conduit à leur redéfinition comme circuits de proximité (Naves, 2016). Dans le même temps, le maraîchage biologique est devenu une figure récurrente de l'action publique locale agricole. Il est en phase avec les recommandations ministérielles de consommation de fruits et légumes, renvoie à des représentations de santé et de fraîcheur et est adapté à des circuits en vogue, telles les AMAP. Par ailleurs, créer une exploitation maraîchère biologique nécessite peu de foncier ${ }^{8}$ et peut donc se faire avec moins de moyens financiers que pour des exploitations de grandes cultures ou d'élevage.

En ciblant le maraîchage en circuits courts de proximité, le plus souvent biologique, les intercommunalités s'inscrivent donc dans un contexte de valorisation d'un modèle agricole atypique, décrit comme vertueux.

\section{Un développement porté par des alliances entre élus, porteurs de projet et acteurs agricoles dominants}

31 Les actions foncières agricoles sont marquées politiquement, portées par une frange particulière d'élus. À Millau et Amiens, les élus impliqués sont premiers vice-présidents d'intercommunalité et ont une orientation militante favorable à l'agriculture paysanne et aux circuits locaux: Slow Food et Europe Écologie - Les Verts (comme dans les cas vauclusien et maralpin étudiés par Jarrige et Napoléone, 2015 et Samak, 2013). À SaintAffrique, c'est la position de marginal-sécant ${ }^{9}$ d'un élu également enseignant au lycée agricole qui a été un élément déclencheur.

La sélection des agriculteurs bénéficiaires est influencée par des relations de proximité entre acteurs publics et porteurs de projet. En effet, contrairement à ce qui est souvent pratiqué (Chavey et Bertrand, 2015 ; Perrin et Nougarèdes, 2015 ; Margetic et al., 2017), un appel à candidatures n'a été organisé que dans un seul des cinq projets (l'espace-test du Saint-Affricain). Les autres collectivités ont proposé des terres soit à des porteurs de projet qu'elles avaient identifiés comme ayant des besoins fonciers, soit à des agriculteurs reconnus. Le cas du Jardin du Chayran illustre cette dimension relationnelle. L'occupation des terres en 2010 est le seul épisode conflictuel dans une relation marquée par une logique « clientélaire » (Guillemin et Walker, 2016) : « le jardin du Chayran est une grosse structure très favorisée : il reçoit beaucoup d'aides, a beaucoup de surfaces, a beaucoup de monde à des coûts salariaux bas $»^{10}$. C'est la seule exploitation subventionnée par la collectivité, pour ses activités de réinsertion, d'approvisionnement des citadins et d'exploration foncière, qui lui valent d'être qualifiée de "partenaire local indispensable par son expérience » (Communauté de communes Millau Grands Causses, 2011). Cette situation s'explique en partie par la grande légitimité de son fondateur, doté d'un fort capital d'autochtonie (Retière, 2013), acquis au cours d'une carrière militante 
dans les champs agricole (vétéran du Larzac), de l'action sociale (fondateur de l'association de réinsertion) et politique (conseiller général socialiste pendant une trentaine d'années, son successeur est l'ancien maire de Millau).

Des acteurs-relais du monde agricole comptent enfin dans le déroulement des projets. La profession agricole instituée (syndicats, Chambres d'agriculture et Safer) peut selon les cas être contournée par l'intercommunalité quand elle s'oppose à ses projets (comme à Brive : Marty, 2014) ou bien constituer un appui, pour résoudre un conflit de légitimité (à Amiens) ou pour accéder à l'information foncière (à Millau et Saint-Affrique). La taille de la ville influe aussi probablement sur la relation entre la collectivité urbaine et la profession agricole: une collectivité de grande taille pèse plus face aux agriculteurs. Quand la ville est petite, les représentants agricoles ont plus de poids: à Millau, l'intercommunalité a laissé la Safer acheter une terre qu'elle était sur le point d'acquérir ; à Amiens, l'intercommunalité a dû faire appel au président de la Chambre d'agriculture pour que les agriculteurs en place acceptent de laisser la place aux nouveaux maraîchers.

Ainsi, les projets des villes intermédiaires sont issus de la rencontre entre des élus de premier plan engagés pour un modèle agricole spécifique, des porteurs de projet et des acteurs-relais du monde agricole. Ces projets se font sans appel à candidatures, ce qui renvoie au caractère récent et exploratoire de ces actions publiques.

\section{Des terres mobilisées par opportunité ou par stratégies d'acquisition}

La mobilisation foncière par les collectivités urbaines prend deux modalités : l'acquisition de terres pour des objectifs alimentaires affichés et la valorisation de réserves foncières réinterprétées comme une ressource nourricière de proximité. Dans le premier cas, les intercommunalités acquièrent des terres via la Safer, comme dans les hortillonnages à Amiens ou dans les fonds de vallées du Sud-Aveyron. Dans le deuxième cas, le plus important en termes de surfaces, des réserves foncières prévues pour des projets d'urbanisation sont remobilisées. Ces terrains publics se retrouvent sans affectation parce qu'un changement d'élus ou une servitude environnementale a remis en cause le projet d'aménagement. Leur réorientation vers l'agriculture est peu coûteuse pour les collectivités. Ce mécanisme, plutôt courant (Marty, 2014 ; Jarrige et Napoléone, 2015), est à l'œuvre dans trois des cinq cas : à Amiens et Saint-Affrique dans une ZAC, à Millau dans un espace naturel sensible en friche. Le caractère parfois inondable des terrains favorise ces changements d'affectation, même s'il est défavorable au maraîchage.

Malgré ces effets d'opportunité, les intercommunalités justifient parfois a posteriori le choix de ces terres. Les périmètres de protection de captage d'eau sont présentés dans la littérature comme un facteur déclencheur d'actions foncières agricoles publiques (Barataud et Hellec, 2015 ; FNAB, 2014 ; Terre de Liens, 2013). Pour améliorer la qualité de l'eau, des collectivités décident de contrôler les pratiques agricoles, notamment par la maitrise foncière, et de favoriser parallèlement l'approvisionnement de la ville-centre. Cependant, dans nos deux cas de protection de captage, les collectivités ont mobilisé cette ressource argumentative a posteriori. Initialement, la mise en friche était considérée comme la meilleure solution pour protéger la ressource en eau. Ce sont les futurs exploitants qui ont proposé de pratiquer le maraîchage biologique. À Millau, les élus ont finalement accepté après avis positif du Ministère de l'Environnement. À Amiens, le soutien de Terre de Liens leur a permis de convaincre l'Agence régionale de la santé, initialement opposée. Ce caractère de ressource argumentative a posteriori ressort 
particulièrement à Amiens, puisque les autres parcelles publiques du périmètre de protection sont toujours cultivées en céréaliculture conventionnelle.

Ainsi, deux types de terres sont mobilisés par les collectivités : des terres acquises pour des fins expressément alimentaires et des terres initialement promises à l'artificialisation, réorientées vers l'agriculture par opportunité. La localisation de ces terres au sein d'un périmètre de protection de captage d'eau potable sert parfois de justification a posteriori.

\section{Les modes de mise à disposition des terres offrent une sécurité inégale aux exploitants agricoles}

Les statuts juridiques des terres mobilisées sont variés : nous proposons de les aborder par les faisceaux de droits de propriété (Schlager et Ostrom, 1992 ; Lavigne Delville, 2010), c'est-à-dire en décomposant les droits détenus sur ce foncier. Ces terres ne sont pas nécessairement des propriétés intercommunales, au sens du droit français. En effet, les droits d'aliénation (c'est-à-dire de vente ou de don des terres) appartiennent dans deux cas à d'autres acteurs publics : un syndicat de ZAC à Amiens et la région Occitanie à SaintAffrique. Les agriculteurs sont, quant à eux, détenteurs exclusifs des droits de production : ils sont les seuls à pouvoir semer, labourer et faire pâturer les terres. Enfin, les droits d'administration sont partagés entre intercommunalités et exploitants: le tableau 2 montre que le choix des types de cultures et des pratiques de production d'une part et le choix des usagers d'autre part n'appartiennent pas aux mêmes acteurs selon les exploitations.

Tableau 2 : Répartition des droits de gestion entre acteurs

\begin{tabular}{|c|c|cc|cc|}
\hline Types & 1 & & 2 & 3 \\
\hline Exploitations & $\begin{array}{c}\text { Exploitations individuelles } \\
\text { des hortillonnages }\end{array}$ & GAEC & $\begin{array}{c}\text { Exploitation } \\
\text { individuelle }\end{array}$ & $\begin{array}{c}\text { Atelier-chantier } \\
\text { d'insertion associatif }\end{array}$ & Espace-test \\
\hline $\begin{array}{c}\text { Choix des cultures et } \\
\text { des pratiques }\end{array}$ & Exploitants & $\begin{array}{c}\text { Préfet, intercommunalité } \\
\text { et exploitants }\end{array}$ & $\begin{array}{c}\text { Intercommunalité et } \\
\text { exploitants }\end{array}$ & $\begin{array}{c}\text { Préfet et } \\
\text { intercommunalité }\end{array}$ & Intercommunalité \\
\hline Choix des usagers & $\begin{array}{c}\text { Exploitants et } \\
\text { intercommunalité }\end{array}$ & $\begin{array}{c}\text { Exploitants et } \\
\text { intercommunalité }\end{array}$ & $\begin{array}{c}\text { Exploitants et } \\
\text { intercommunalité }\end{array}$ & Intercommunalité & $\begin{array}{c}\text { Intercommunalité et } \\
\text { comité de pilotage }\end{array}$ \\
\hline
\end{tabular}

Le premier type correspond à la répartition (très courante en France) définie par le statut du fermage : les exploitants sont libres du choix de leurs pratiques et peuvent céder leur bail dans le cadre familial, sans avoir à demander l'accord du propriétaire. Il s'agit ici d'exploitations familiales qui se sont agrandies grâce à l'intervention foncière publique. Le deuxième type, également en fermage, se différencie du premier par un partage des droits sur les cultures et les pratiques. Un document réglementaire (arrêté préfectoral) ou un contrat (bail à clauses environnementales) imposent à l'exploitant de suivre le cahier des charges de l'agriculture biologique. Les exploitations ont ici été créées suite à l'intervention foncière publique, par des néo-agriculteurs. Dans le troisième type, les intercommunalités détiennent tous les droits d'administration, parfois secondées par d'autres acteurs: les exploitants doivent cultiver les terres en maraîchage biologique et leurs droits d'usage sont limités dans le temps. À Millau, le Jardin de Chayran bénéficie d'une convention d'occupation temporaire et précaire, renouvelable chaque année. Au sein de l'espace-test de Saint-Affrique, les futurs maraîchers sont employés en contrats d'aide au projet d'entreprise, d'une durée maximale de trois ans. Cet éventail de statuts juridiques se traduit par une précarité foncière variable. La précarité la plus faible est assurée par le statut du fermage (type 1). Elle est la plus forte dans des formes de 
délégation s'approchant de la régie agricole, où l'intercommunalité contrôle de nombreux droits fonciers (type 3). Entre les deux, le fermage intégrant des enjeux environnementaux émerge depuis une décennie (type 2).

Cette diversité de statuts juridiques renvoie à des représentations du foncier et de l'activité agricole en concurrence, opposant les collectivités urbaines et la profession agricole. Les syndicats agricoles majoritaires FNSEA ${ }^{11}$ et JA ${ }^{12}$ désapprouvent les actions foncières publiques. Un rapport d'orientation de JA est particulièrement clair à ce sujet (Daurelle et al., 2015) : le modèle d'exploitation familiale ${ }^{13}$ et la liberté de choix des pratiques (notamment vis-à-vis de l'environnement) y sont défendus contre la propriété publique du foncier agricole (p. 42). Celle-ci est critiquée du fait des restrictions liées aux baux ruraux environnementaux et à l'agriculture biologique, ainsi que des dépenses engagées, jugées excessives. La régie publique y est décrite comme la pire situation, «l'entreprise agricole [étant] par nature une entreprise privée» (p. 44). À Millau, un représentant de JA s'est opposé au principe d'installation agricole par un acteur public: « il y a un truc qui me dérange, c'est que la collectivité se positionne pour acheter du foncier et installer des agriculteurs dessus : [...] ce n'est pas son boulot. ${ }^{14}$. Parce qu'il ne l'aurait pas achetée, le futur exploitant travaillerait mal la terre. À Amiens, l'attachement à la propriété foncière est plus faible, mais certains agriculteurs se sont aussi opposés aux installations maraîchères sur des terres publiques (qu'ils exploitaient auparavant), craignant que la collectivité n'installe à leur détriment d'autres agriculteurs sur le reste de ses importantes réserves foncières.

Ainsi, les intercommunalités font exploiter leurs terres selon trois modalités, correspondant à différents niveaux de contrôle des types de culture et de pratiques. Le syndicat majoritaire s'oppose à ce changement de statut juridique du foncier agricole, qui s'accompagne de la promotion d'un modèle agricole "atypique", le maraîchage biologique en circuit court de proximité. Qu'elles acquièrent ces terres expressément ou qu'elles les remobilisent pour des fins de relocalisation alimentaire, les collectivités ébauchent à partir d'elles des constructions symboliques du foncier agricole comme ressource nourricière locale.

\section{Une construction incomplète des terres agricoles comme ressources nourricières de proximité}

42 Un décalage existe entre les ambitions de relocalisation alimentaire des collectivités urbaines et leurs réalisations foncières. Il tient en partie au manque de compétences et de légitimité des intercommunalités sur les questions agricoles.

\section{Un décalage entre les intentions des documents de planification et les réalisations}

Les Schémas de cohérence territoriale (SCoT) et Plans locaux d'urbanisme (PLU) donnent des indications sur les représentations et stratégies des villes intermédiaires en matière agricole. Comme le souligne Guiomar (2011), « un PLU ou un SCoT bien abouti pourra préciser les orientations agricoles prioritaires [...], par exemple les productions vivrières ou identitaires, ce qui pourra [...] se traduire par une protection renforcée des espaces les plus propices à ces productions ». 
Adopté en 2012, le SCoT du Grand Amiénois promeut une agriculture compétitive, intégrée aux industries. Il encourage toutefois les communes et intercommunalités à « faciliter le développement du maraîchage par la mise à disposition de terrains agricoles dont elles ont la maîtrise foncière, en particulier dans le fond de vallée [...] et autour des captages d'eau potable» (Aduga, 2012), et souligne l'importance du développement des circuits courts. Pour Amiens Métropole en particulier, le SCoT promeut des couronnes «d'agriculture nourricière » autour des villages, produisant fruits et légumes au plus près des habitants. Les élus n'ont pas donné suite à ces prescriptions, si ce n'est pour l'installation des deux maraîchers dans la ZAC. Le conseil municipal concerné a modifié son PLU afin d'autoriser les serres-tunnels nécessaires à cette activité. Ce faisant, il affirme vouloir « recréer une zone de production de fruits et légumes dans la métropole amiénoise [...] et relocaliser au maximum les modes de commercialisation » (Commune de Pont-de-Metz, 2011).

Adopté en 2016, le SCoT du Sud-Aveyron (incluant les intercommunalités de Millau et Saint-Affrique) cite davantage l'agriculture. Il appelle au maintien des filières de qualité et promeut la diversification des productions. Il encourage les municipalités à protéger dans les PLU les zones de maraîchage (entre autres), en soulignant qu'elles « sont pour l'heure difficilement mobilisables [et que] des projets de relance en marche depuis plusieurs années doivent être soutenus » (PNR des Grands Causses, 2016). La commune de Millau avait déjà tenté en 2010 de favoriser le maraîchage dans son PLU, en créant des zones formellement dédiées à cette production. Elles ne couvrent toutefois que 14 ha, en zone inondable, pâturés par du bétail. Les meilleures terres maraîchères de la commune, mises à disposition du Jardin du Chayran, sont classées en zone naturelle, ce qui empêche l'installation de serres.

Un décalage apparaitt ainsi entre les intentions formulées par les collectivités urbaines en termes d'action foncière pour la relocalisation alimentaire et les réalisations effectives. Paradoxalement, c'est à Amiens que la relocalisation alimentaire est le plus explicitement présentée comme devant faire l'objet d'une action foncière, alors qu'elle y est bien plus difficilement réalisable que dans le Sud Aveyron. Le foncier est rarement qualifié comme ressource nourricière locale en-dehors des documents de planification. Sur les parcelles concernées, aucune affiche ne mentionne par exemple leur statut juridique et symbolique spécifique. La seule ferme à avoir été officiellement inaugurée (à Amiens) l'a été à l'initiative de Terre de Liens, qui avait accompagné le projet.

\section{Les collectivités urbaines manquent encore de compétences et de légitimité sur les questions agricoles}

47 Les collectivités peinent à construire concrètement et symboliquement les terres agricoles comme ressources nourricières de proximité parce qu'elles se heurtent à trois difficultés.

La première difficulté est liée à des freins juridiques et économiques. Les SCoT et PLU ne peuvent pas soutenir par des dispositions réglementaires un modèle agricole. Dépourvues d'instrument pour influencer les productions, pratiques et modes de commercialisation, les collectivités peuvent seulement acquérir des terrains ou chercher à établir des conventions (chartes). Pour acheter du foncier agricole, l'expropriation pour cause d'utilité publique n'est utilisée que sur les périmètres de protection de captage d'eau potable. Les collectivités doivent sinon préempter, mais très peu de terres se vendent ${ }^{15}$. 
Une fois les terres acquises, elles doivent installer un agriculteur adhérant au modèle agricole choisi. Si un fermier est en place, son éviction ne peut se faire qu'au prix d'indemnisations. Enfin, le maintien d'un modèle agricole n'est contractuellement possible que pour l'agriculture biologique (par un bail à clauses environnementales).

Ensuite, les collectivités urbaines manquent parfois de légitimité et de soutiens pour défendre l'agriculture nourricière de proximité. Elles apparaissent certes comme de nouveaux acteurs normatifs en matière agricole (Marty, 2014), mais elles ont en fait une position marginale. Elles manquent encore de légitimité dans le domaine agricole, même si elles siègent dans certaines instances de décision (Comités techniques départementaux des Safer, Commissions départementales d'orientation de l'agriculture des préfectures). De plus, leur approche alimentaire, transversale et territoriale, est soutenue par des franges minoritaires et dominées du secteur agricole : la Confédération paysanne, le pôle InPACT ${ }^{16}$ et des organisations de la société civile ${ }^{17}$. Elle ne s'impose pas face à l'approche sectorielle et corporatiste des organisations agricoles majoritaires (Thareau, 2011 ; Blanc, 2012; Barral et Pinaud, 2015). Barthès et Bertrand (2016) l'illustrent à partir de la politique d'installation en agriculture biologique et en circuit courts menée à Voiron. Les exploitants y pointent le risque d'une agriculture "administrée ", déconnectée des réalités professionnelles et répondant aux aspirations de la seule nouvelle petite bourgeoisie intellectuelle urbaine. En effet, les groupes sociaux (tant urbains qu'agricoles) soutenant l'inscription à l'agenda politique local des actions foncières sont limités et mobilisent souvent un registre très politisé des « alternatives » agricoles et alimentaires. Faute de revendication à la fois explicite et multifactorielle des projets par les élus, les collectivités peuvent manquer de soutiens pour faire aboutir de tels projets. réducteur par rapport au projet global de relocalisation alimentaire. Ce ciblage ne permet de répondre qu'à une petite partie des besoins alimentaires des citadins. Il exclut les autres productions et la plupart des terres agricoles d'une construction comme ressources nourricières. Les actions foncières sont donc d'envergure limitée, et bien éloignées d'autres grands projets publics sur le foncier agricole tels que les réformes agraires. Ensuite, les exploitations maraîchères biologiques en circuits courts de proximité sont des systèmes complexes (Dufour et Lanciano, 2012). Les collectivités urbaines qui les promeuvent n'intègrent pas toujours leurs contraintes en termes de viabilité économique et sociale (organisation et pénibilité du travail, nécessité de polyvalence, gestion des aléas, faible rémunération horaire du travail), ce qui peut impacter la pérennité à long terme des installations.

51 Ainsi, les difficultés de construction des terres agricoles comme ressources nourricières de proximité s'expliquent (i) lors de la mise en œuvre des projets, par le recours nécessaire à des outils juridiques importés d'autres champs de l'action publique, (ii) en amont, par le manque de légitimité agricole des collectivités et par une structuration insuffisante des soutiens sociaux aux projets et (iii) en aval, par le ciblage sur une production à l'emprise foncière limitée et impliquant des conditions de travail difficiles.

\section{Conclusion}

52 Cet article a proposé une analyse des ressorts, implications et limites des actions foncières de villes intermédiaires en faveur de la relocalisation alimentaire. Les régularités qui ressortent peuvent former une grille de lecture pour comprendre d'autres 
trajectoires. Les actions étudiées sont récentes, exploratoires, et souvent temporaires. Elles s'appuient sur des préoccupations initialement non foncières et ciblent un modèle agricole atypique, le maraîchage biologique en circuits courts de proximité. Les projets résultent de la rencontre entre élus de premier plan, porteurs de projet et acteurs dominants du monde agricole. Le choix des bénéficiaires est marqué par des logiques clientélaires. Les terres sont soit mobilisées suite à l'annulation de projets d'urbanisation, soit acquises suite à une veille foncière. Les sols favorables au maraîchage sont recherchés a priori, tandis que la localisation dans des périmètres de captage d'eau potable sert de ressource argumentative a posteriori. Enfin, le contrôle des intercommunalités sur le choix des pratiques et productions est souvent fort et inhabituel pour les agriculteurs accoutumés au fermage. Ces actions foncières publiques sont critiquées par les secteurs majoritaires de la profession agricole. Ainsi, le statut public de ces «terres nourricières » n'est que très rarement mis en avant par les intercommunalités.

Les trois intercommunalités de villes intermédiaires étudiées ne représentent probablement pas la diversité des trajectoires possibles. Si le cas d'Albi, évoqué en introduction, apparaît en phase avec des points évoqués (maraîchage biologique en circuit court de proximité, terres alluvionnaires et inondables), la politique foncière y est revendiquée et les terres ciblées sont présentées comme le nouveau cœur nourricier de la ville. Son développement peut donc être intéressant à suivre, notamment en ce qui concerne la construction du foncier comme ressource nourricière: le fait qu'il ait été impulsé par une équipe élue en 2014 pourrait indiquer l'émergence d'une nouvelle génération de " retour à la terre » des villes intermédiaires. Leurs actions pourraient ainsi se rapprocher progressivement de celles des métropoles, où les politiques foncières agricoles apparaissent jusqu'à présent plus cohérentes, pérennes et revendiquées, et concernent des surfaces souvent plus importantes.

En nous concentrant sur les exploitations agricoles, notre analyse offre ainsi une perspective originale sur la mise en œuvre du locavorisme par l'amont de la chaîne alimentaire - angle souvent délaissé au profit des approches centrées sur la consommation. Ce faisant, elle laisse toutefois de côté des politiques plus sociales, comme celle de Roanne, dont les actions foncières ciblent des jardins et vergers associatifs ou collectifs (Delfosse, 2015).

En définitive, l'article souligne les rapports différenciés qu'entretiennent les collectivités urbaines et le monde agricole à la terre agricole. Les acteurs publics urbains en ont une représentation idéalisée, marquée par la "promesse de différence» des modèles alternatifs (Le Velly, 2017). De fait, les trois intercommunalités étudiées ne reconnaissent pas la diversité des modèles agricoles en présence, leur complexité et leur utilité. Elles manquent de compétences et de légitimité agricoles, leurs politiques foncières découlant d'ailleurs d'autres enjeux (paysage, emploi, santé). Elles ont aussi moins conscience de la finitude de la ressource foncière, car elles cherchent encore à se doter des équipements $\mathrm{du}$ niveau des grandes villes. L'agriculture et l'alimentation sont alors mises au service d'une politique urbaine en phase avec leur électorat, notamment avec la nouvelle petite bourgeoisie intellectuelle citadine. Les volontés urbaines sont donc à la fois symboliques et relativement opportunistes.

L'inscription des villes intermédiaires dans le mouvement de relocalisation alimentaire reste anecdotique en surface et se limite à son versant technique et opérationnel. Elle s'appuie peu sur les rhétoriques de la souveraineté et de l'autonomie alimentaires, souvent mobilisées par la société civile. C'est que la propriété foncière ne s'inscrit pas 
dans un discours explicitement politique. Elle pourrait être conçue comme une communalisation des moyens de production alimentaire et viser la sortie de la terre agricole de l'économie de marché, ainsi que sa politisation comme commun nourricier des citadins. En combinant des politiques alimentaires et foncières, les villes seraient alors à même de fonder, comme une critique « alternative » en actes, le projet global de démocratie alimentaire (Collard-Dutilleul, 2013).

Malgré des alliances sociales inachevées, un cadre juridique restrictif et les contractions budgétaires, les actions étudiées peuvent constituer un premier pas. D'abord, le regain d'intérêt des intercommunalités de villes intermédiaires pourrait s'appuyer plus systématiquement sur des secteurs sociaux multi-situés, qui tissent des liens entre ville et agriculture. Les « néo-paysans $\aleph^{18}$ s'installant en nombre croissant sont souvent d'anciens citadins et pourraient endosser ce rôle de passeurs. Un autre vecteur pourrait être le mouvement Terre de Liens, qui recherche des coopérations renforcées avec les collectivités et a progressivement établi des ponts avec certains acteurs dominants du monde agricole. Ensuite, des moyens juridiques adéquats pourraient être mobilisés pour mener les actions foncières : des déclarations d'utilité publique pourraient être arrêtées par les préfets à la demande des intercommunalités portant des projets fonciers pour la relocalisation alimentaire. Enfin, comme l'avait envisagé Pisani (1977), une réforme de la taxe foncière permettrait d'affecter des budgets fléchés à des actions foncières publiques agricoles.

\section{BIBLIOGRAPHY}

Aduga, 2012, Schéma de Cohérence Territoriale du pays du Grand Amiénois, Amiens, SCoT

ARNAL C., 2012a, La place et le rôle de l'agriculture à la périphérie des villes moyennes. Le cas des villes d'Annecy, Bourg-en-Bresse, Montbrison et Romans-sur-Isère, thèse de doctorat de géographie, Université Lumière Lyon 2.

ALLENS G. et LECLAIR L., 2016, Les néo-paysans, Paris, Seuil et des « Assises néo-paysannes » (première édition en 2017).

ARNAL C., 2012b, L'agriculture, élément de la qualité de vie des villes moyennes, Revue d'Économie Régionale et Urbaine, $\mathrm{n}^{\circ}$ 2012/2, p. 245-264.

BARATAUD F. et HELLEC F., 2015, L'outil foncier, une solution délicate pour protéger les captages d'eau potable, Économie rurale, $\mathrm{n}^{\circ} 347$, p. 3-20.

BARRAL S. et PINAUD S., 2015, Les agriculteurs, maîtres tenaces de l'accès à la terre. Les impasses de la politique foncière française contemporaine, Mouvements, $n^{\circ} 84$, p. 64-72.

BARTHÈs C. et BERTRAND N., 2016, L'échelon intercommunal dans la gouvernance foncière des espaces agricoles, Économie rurale, $\mathrm{n}^{\circ}$ 353-354, p. 65-79.

BERROIR S., CATTAN N., DOBRUSZKES F., GUÉROIS M., PAULUS F., VACCHIANI-MARCUZZO C., 2017, Les systèmes urbains français : une approche relationnelle, Cybergeo : European Journal of Geography, DOI :

10.4000/cybergeo. 27945 
BLANC J., 2012, Construire l'alternative agro-alimentaire : ressorts sociaux et politiques du déploiement des AMAP en Île-de-France, Norois, $\mathrm{n}^{\circ}$ 224, p. 21-34.

BRAND C., 2015, Alimentation et métropolisation : repenser le territoire à l'aune d'une problématique vitale oubliée, thèse de doctorat de géographie, Université Grenoble Alpes.

CARCENAC P., 2016, Comment la ville d'Albi veut conquérir son autosuffisance alimentaire, Le Figaro, 14 avril 2016.

CARRIER M., DEMAZIÈRE C., 2012, La socio-économie des villes petites et moyennes : questions théoriques et implications pour l'aménagement du territoire, Revue d'économie régionale et urbaine, $n^{\circ}$ 2012/2, p. 135-149.

Chavey A. et Bertrand J., 2015, Projet d'installation agricole dans la Vallée de la Peyne, colloque ASRDLF « Territoires méditerranéens, agriculture, alimentation et villes », Montpellier, 7-9 juillet 2015.

COLLARD-DUTILlEUL F. éd., 2013, Penser une démocratie alimentaire, San José, INIDA.

Communauté de communes Millau Grands Causses, 2011, Compte-rendu détaillé du conseil de la communauté du 17 février 2011, Délibération du conseil communautaire

Commune de Pont-de-Metz, 2011, Modification du PLU dans le cadre du projet d'agriculture bio sur les terrains de Borealia, Délibération du conseil municipal

Daurelle A., Richard S., Rihouet F., QUeSNel R., DE BoyeR D’Eguiles C., 2015, Foncier : entre avenir et héritages, Paris, Rapport d'orientation présenté au congrès de Jeunes Agriculteurs, 16-18 juin, Le Mans.

DELÉAGE E., 2011, Les mouvements agricoles alternatifs, Informations sociales, n¹64, p. 44-50.

DeLFosSE C., 2011, La patrimonialisation des produits dits de terroir [en ligne], Anthropology of food, $\mathrm{n}^{\circ} 8$, [URL : http://aof.revues.org/6772], consulté le 6 juillet 2017.

DELFosSE C., 2015, Villes moyennes et produits de terroir. Quatre villes à l'ombre de la métropole lyonnaise, in P. MEYZIES et C. MARACHE éd., Produits de terroir. L'empreinte de la ville, Rennes, PUR, p. 259-278.

DEMAZIÈRE C., SERRANO J., VYE D., 2012, Les villes petites et moyennes et leurs acteurs : regards de chercheurs, Norois, $\mathrm{n}^{\circ} 223$, p. 7-10

DUfOUR A. et LANCIANO E., 2012, Chapitre 5. Diversité des trajectoires en maraîchage, in A.-H. PRIGENT-SIMONIN et C. HERAULT-FOURNIER éd., Au plus près de l'assiette, Versailles, Quæ, p. 103-116.

DuVERnoy I., JARRige F., MOUSTIER P., SERRANO J., 2005, Une agriculture multifonctionnelle dans le projet urbain : quelles reconnaissances, quelle gouvernance ?, Les Cahiers de la multifonctionnalité, $\mathrm{n}^{\circ} 8$, p. 87-104.

Fédération nationale d'agriculture biologique des régions de France, 2014, Communauté d'agglomération Seine-Eure et le projet des Hauts-Prés [en ligne], Paris, Fiche-expérience $n^{\circ} 6 \mathrm{du}$ programme « Agriculture biologique et développement local : une boîte à outils pour les collectivités territoriales », [www.devlocalbio.org], consulté le 6 juillet 2017.

Fédération nationale des Safer, 2014, Le prix des terres. Analyse des marchés fonciers ruraux, Paris, $136 \mathrm{p}$.

FÉRÉROL M.-È., 2013. Le modèle de Christaller et les espaces interstitiels du Massif central, M@ppemonde, 112, http://mappemonde.mgm.fr/num40/articles/art13402.html. 
GALLIEN M., 2011, Les représentations et perceptions de la nature dans une ville moyenne. Le cas des marais de l'Yère et de la Voiselle à Bourges, mémoire de master de géographie, Université Lumière Lyon 2

GUILLEMIN P. et WALKER E., 2016, Une action publique « ordinaire »? Réflexion sur la production de l'espace : rapports aux institutions locales et classe de l'encadrement, ESO Travaux et Documents, $\mathrm{n}$ - 40, p. 49-58.

GUIOMAR X., 2011, Les collectivités locales à la recherche d'une agriculture de proximité, POUR, n 209-210, p. 169-183

GuIOMAR X., 2014, La mise en proximité de l'agriculture (péri)urbaine par les collectivités, POUR, $\mathrm{n}^{\circ} 224$ Sociologie des systèmes alimentaires alternatifs, p. 415-426

JARRIGE F. et NAPOLÉONE C., 2015, Comment maintenir des activités agricoles durables face à la pression d'urbanisation? Une politique locale à l'épreuve dans le Vaucluse, in G. VIANEY, M. REQUIER-DESJARDINS et J.-C. PAOLI éd., Accaparement, action publique, stratégies individuelles et ressources naturelles : regards croisés sur la course aux terres et à l'eau en contextes méditerranéens, Montpellier, CIHEA, p. 325-338.

JARRIGE F. et PERRIN C., 2017, L'agriparc : une innovation pour l'agriculture des territoires urbains ?, Revue d'économie régionale et urbaine, $\mathrm{n}^{\circ}$ 2017/3, p. 537-562.

LAVIGNe Delville P., 2010, Sécurisation foncière, formalisation des droits, institutions de régulation foncière et investissements. Pour un cadre conceptuel élargi, Revue des questions foncières, $\mathrm{n}^{\circ} 1$, p. 5-33.

LÉGER-BOSCH C. et ALAVOINE-MORNAS F., 2013, Exploitation agricole périurbaine cherche reconnaissance et visibilité foncière, Géographie et cultures, p. 37-55.

LÉGER-BOSCH C., 2015, Les opérations de portage foncier pour préserver l'usage agricole. Une analyse par les coordinations, les transactions et les institutions, thèse de doctorat d'économie, Université Grenoble Alpes.

LEVESQUE R., LIORIT D., PATHIER G., 2011, Les marchés fonciers ruraux régionaux entre dynamiques des exploitations agricoles et logiques urbaines, Economie et statistique, $\mathrm{n}^{\circ} 444-445$, p. 75-98.

LE VELLY R., 2017, , Paris, Presses des Mines, 200 p.

MARGETIC C. et CHAUCESSE J.-N., 2012, La politique de reconquête des friches agricoles dans l'agglomération nantaise, Cahiers nantais, n² 2012/2, p. 59-68.

MARGETIC C., RougEt N., Schmitt G., 2017, Le foncier agricole à l'épreuve de la multifonctionnalité : desseins environnementaux et alimentaires dans les métropoles lilloise et nantaise, Norois, $\mathrm{n}^{\circ} 241$, p. 87-104

MARTY P., 2014, Les appropriations urbaines de la question agricole. Le cas de Brive, de 1945 à 2012, thèse de doctorat de géographie, Université Paris I Panthéon-Sorbonne.

NAVES P., 2016, L'encadrement des circuits courts. Du secteur agricole aux territoires ?, Économie rurale, $\mathrm{n}^{\circ} 355$, p. 3-19.

PARANTHOËN J.-B., 2015, L'incursion des scientifiques dans l'organisation des marchés agricoles, Politix, $\mathrm{n}^{\circ} 111$, p. 119-140.

PerRin C. et Nougarèdes B., 2015, " Justice issues in farmland protection policies on the urban fringe », colloque international "Agriculture in an Urbanizing Society”, Rome, 14-17 septembre 2015. PISANI E., 1977, Utopie foncière : l'espace pour l'homme, Paris, Gallimard, 212 p. 
Poulot M., 2012, Vous avez dit « locavore » ? De l'invention du locavorisme aux Etats-Unis, Pour, $\mathrm{n}^{\circ} 215-216$, p. 349-354.

Poulot M., 2014, Agriculture et acteurs agricoles dans les mailles des territoires de gouvernance urbaine : nouvelle agriculture, nouveaux métiers? Espaces et sociétés, $\mathrm{n}^{\circ} 158$, p. 13-30.

PNR des Grands Causses, 2016, Schéma de Cohérence Territoriale du Parc naturel régional des Grands Causses, Millau, SCoT

RETIÈRE J.-N., 2013, Capital d'autochtonie, in CASILLO I., BARBIER R., BLONDIAUX L., CHATEAURAYNAUD F., FOURNIAU J.-M., LEFEBVRE R., NEVEU C. et SALLES D. éd., Dictionnaire critique et interdisciplinaire de la participation, Paris, GIS Démocratie et Participation, [http:// www.dicopart.fr/it/dico/capital-dautochtonie], consulté le 6 juillet 2017.

RIEUTORT L., 2018, Introduction, p. 5-8, actes du colloque « Métropoles, villes intermédiaires et ruralités. Quelles interactions au service du développement territorial ? ", Clermont-Ferrand, 23-24 novembre 2017 [http://iadt.fr/uploads/ressource/html/25/IADT_CollMetropoles_ActesNumeriques.pdf]

SAMAK M., 2013, Quand la « bio » rebat les cartes de la représentation des agriculteurs. L'institutionnalisation de l'agriculture biologique dans les Alpes-Maritimes, Politix , n 103, p. 125-148.

SCHLAGER E. et OSTROM E., 1992, Property-Rights Regimes and Natural Resources: A Conceptual Analysis, Land Economics, Vol. 68, n 3, p. 249-262.

Terre de Liens, 2013, Agir sur le foncier agricole. Un rôle essentiel pour les collectivités locales, Crest, Guide pratique, $136 \mathrm{p}$.

THAREAU B., 2011, Réguler l'accès à la terre, la réinvention locale du corporatisme agricole, thèse de doctorat en sociologie, Université Paris Ouest Nanterre La Défense.

THAREAU B. et FABRY M., 2013, Actions foncières au nom de l'environnement : des élus locaux interviennent dans l'évolution de l'agriculture, in BERTRAND N. éd., Terres agricoles périurbaines. Une gouvernance foncière en construction, Versailles, Quae, p. 135-153.

\section{NOTES}

1. Directeurs généraux des services

2. Société d'aménagement foncier et d'établissement rural

3. Entretien avec un chargé de mission, mars 2016

4. Zone d'aménagement concerté

5. Entretiens avec la vice-présidente de l'époque, février 2014 puis janvier 2016.

6. En 1971, le Ministère de la Défense annonce l'extension du camp militaire du Larzac sur 14000 ha de terres agricoles et de bois : plus de cent exploitants concernés s'y opposent, notamment par le squat de fermes acquises par l'État, jusqu'à l'abandon du projet en 1981.

7. D'après le site du Réseau national des espaces-tests agricoles (https://reneta.fr/Lesmembres-du-RENETA, consulté le 17 avril 2018).

8. En moyenne entre 1 et 2 ha par emploi.

9. Acteur situé au croisement de plusieurs champs, qui joue un rôle d'interprète.

10. Entretien avec une maraîchère, février 2014.

11. Fédération nationale des syndicats d'exploitants agricoles

12. Jeunes Agriculteurs

13. Où le foncier, le capital et le travail sont entre les mains d'une même famille 
14. Entretien téléphonique, février 2016.

15. En France, un terrain agricole est vendu en moyenne une fois tous les cent ans s'il est loué, une fois tous les cinquante ans s'il est exploité par son propriétaire (Fédération nationale des Safer, 2014, p. 10).

16. "Initiatives pour une agriculture citoyenne et territoriale ", qui rassemble des mouvements de développement agricole et rural alternatifs (Déléage, 2011).

17. Dont Attac, Alternatiba, Biocoop ou Greenpeace, associés à la campagne «Produire à quel prix, manger à quel prix ", en 2016 et 2017, ou le Miramap, avec sa campagne « Nous produisons / mangeons / décidons. Ensemble pour une démocratie agricole et alimentaire », en 2017.

18. Terme repris de l'ouvrage D'ALLENS G. et LECLAIR L., 2016.

\section{ABSTRACTS}

In France, there is a growing interest of urban local authorities for their neighboring farmlands: to strengthen their local food system, they make farmers farm lands they own. How do they come to implement such actions? Which types of land, farmers and farming styles are targeted? We carried out a qualitative research in the urban agglomerations of Amiens (in the Picardy), Millau and Saint-Affrique (both in Mediterranean mountains). These urban trajectories have little strategic direction. They target market gardening and short supply chains. This farming style is set up on farmlands which legal, physical and symbolical statuses are specific. A diversity of land tenure forms is used by the local authorities. It signals how conflicting legitimacies are assigned to farmland in relation to local food provisioning.

En France, des intercommunalités de villes intermédiaires portent un intérêt nouveau aux terres agricoles de leur périphérie. Pour renforcer leur système alimentaire local, elles font exploiter du foncier public. Comment en arrivent-elles à mettre en œuvre de telles actions? Quels types de terres, d'agriculteurs et de modèle agricole ciblent-elles? Nous donnons un aperçu de trois trajectoires urbaines, à partir d'enquêtes qualitatives dans les intercommunalités d'Amiens, Millau et Saint-Affrique. Peu stratégiques, elles ciblent le maraîchage en circuit court et cherchent à l'implanter sur des terres aux statuts juridiques, physiques et symboliques particuliers. Les différentes formes de mise à disposition renvoient à des légitimités en conflit du rôle des terres dans l'approvisionnement alimentaire local.

\section{INDEX}

Keywords: farmlands, market gardening, proximity farming, public policy, relocalized food system, short food supply chains

Mots-clés: agriculture de proximité, circuits courts, foncier agricole, maraîchage, politique publique, système alimentaire relocalisé 


\section{AUTHORS}

\section{ADRIEN BAYSSE-LAINÉ}

Université Lumière Lyon 2 - EA Laboratoire d'Études Rurales. 16 avenue Berthelot, 69007 Lyon, France / INRA-SAD - UMR Innovation. 2 place Pierre Viala, bât. 27, 34060 Montpellier Cedex 02, France abl@posteo.eu

\section{COLINE PERRIN}

INRA-SAD - UMR Innovation. 2 place Pierre Viala, bât. 27, 34060 Montpellier Cedex 02, France

\section{CLAIRE DELFOSSE}

Université Lumière Lyon 2 - EA Laboratoire d'Études Rurales. 16 avenue Berthelot, 69007 Lyon, France claire.delfosse@wanadoo.fr 